BNL-112678-2016-JA

\title{
Nanowire growth kinetics in aberration corrected environmental transmission electron microscopy
}

Yi-Chia Chou, Federico Pancierac,

Mark C. Reuter, Eric A. Stach, and Frances M. Ross

Submitted to the ChemCatChem

March 2016

Center for Functional Nanomaterials

Brookhaven National Laboratory

\author{
U.S. Department of Energy \\ USDOE Office of Science (SC), \\ Basic Energy Sciences (BES) (SC-22)
}

Notice: This manuscript has been authored by employees of Brookhaven Science Associates, LLC under Contract No. DE-SC0012704 with the U.S. Department of Energy. The publisher by accepting the manuscript for publication acknowledges that the United States Government retains a non-exclusive, paid-up, irrevocable, world-wide license to publish or reproduce the published form of this manuscript, or allow others to do so, for United States Government purposes. 


\section{DISCLAIMER}

This report was prepared as an account of work sponsored by an agency of the United States Government. Neither the United States Government nor any agency thereof, nor any of their employees, nor any of their contractors, subcontractors, or their employees, makes any warranty, express or implied, or assumes any legal liability or responsibility for the accuracy, completeness, or any third party's use or the results of such use of any information, apparatus, product, or process disclosed, or represents that its use would not infringe privately owned rights. Reference herein to any specific commercial product, process, or service by trade name, trademark, manufacturer, or otherwise, does not necessarily constitute or imply its endorsement, recommendation, or favoring by the United States Government or any agency thereof or its contractors or subcontractors. The views and opinions of authors expressed herein do not necessarily state or reflect those of the United States Government or any agency thereof. 


\title{
Nanowire growth kinetics in aberration corrected environmental transmission electron microscopy
}

\author{
Yi-Chia Chou ${ }^{a, b, c, *}$, Federico Panciera ${ }^{c, d}$, Mark C. Reuter ${ }^{c}$, Eric A. Stach ${ }^{a}$ and Frances M. Ross, ${ }^{c, *}$
}

\begin{abstract}
We visualize atomic level dynamics during Si nanowire growth using aberration corrected environmental transmission electron microscopy, and compare with lower pressure results from ultrahigh vacuum microscopy. We discuss the importance of higher pressure observations for understanding growth mechanisms and describe protocols to minimize effects of the higher pressure background gas.
\end{abstract}

Semiconductor nanostructures present exciting possibilities for electronic and energy applications. In particular, catalytic growth of $\mathrm{Si}$, Ge and III-V materials by the vapor-liquid-solid (VLS) or vaporsolid-solid (VSS) mechanisms can produce nanowires with morphology, dimensions, and electronic properties that are potentially suitable for deep scaling of electronic devices, as well as applications in sensors, batteries and photovoltaics. From both a scientific and technological perspective, precise control of the final structure formed by the VSS and VLS processes requires an understanding of the atomic-level details of growth.

In VLS and VSS growth, the source material is supplied from a gas phase precursor, dissolves into nanoscale catalysts and precipitates at the catalyst/substrate interface. The catalyst is liquid or solid for VLS and VSS growth, respectively. For Si and Ge, both VLS and VSS processes have been examined using in situ electron microscopy ${ }^{1-13}$; see the fuller list of citations in Ref. 1 . In situ observations show, for example, the VLS process by which liquid eutectic droplets of $\mathrm{AuSi}$ form when $\mathrm{Au}$ is deposited on $\mathrm{Si}$, and their subsequent catalytic action in forming Si nanowires. ${ }^{1-5} \mathrm{Si}$ with $\mathrm{Al}^{6}$ and Ge with $\mathrm{Au}^{7}$ are other examples of VLS systems. VSS growth has been imaged in situ using other catalytic metals: $\mathrm{Cu}^{8}$ and $\mathrm{Pd}^{9}$ form solid silicides that catalyze Si nanowire growth, while $\mathrm{AuAg}^{10}$ or $\mathrm{AuAl}^{11}$ alloys can form either solid or liquid catalysts, depending on the temperature and alloy composition. The in situ experiments show that in all cases Si grows by repeated nucleation and flow of $0.3 \mathrm{~nm}$ height (atomic bilayer) steps at the catalyst/nanowire interface ${ }^{1,9}$; growth models derived from in situ observations explain step flow kinetics and interface morphology ${ }^{12,13}$ and the effect of catalyst phase on compositional profiles, ${ }^{10,11}$ helpful in designing electronic device structures.

The majority of these in situ observations have been made under ultra-high vacuum (UHV) conditions ${ }^{1-4,6-8,10-13}$. UHV microscopy (UHV-TEM) offers clean surfaces and well-controlled growth kinetics due to the low background pressure, $\sim 10^{-10}$ Torr. ${ }^{1}$ However, vacuum design also restricts the pressure during growth to below $~$ $10^{-5}$ Torr. In conventional reactors, Si and Ge (and III-V) nanowires are grown at higher pressures and not under UHV conditions. ${ }^{14,15}$ This means that there is a "pressure gap" between the conditions used for microscopy observation and the conditions for real-life growth. Such a pressure gap is familiar in catalysis studies ${ }^{16}$ and has been narrowed, if not completely closed, through the use of environmental TEM (ETEM). ${ }^{17}$ ETEM offers a higher pressure during imaging, up to several Torr, but with a base pressure, $\sim 10^{-6}$ Torr, that is not as low as for UHV-TEM. Some ETEMs also provide the capability of aberration-corrected imaging, with the exciting prospect of growth information with higher spatial resolution ${ }^{18}$ than available from either conventional ETEM or UHV-TEM. Furthermore, several modern detector designs ${ }^{19}$ can provide temporal resolution of several hundred images per second, an improvement over the 30 fps typically obtained in UHV-TEM or conventional ETEM, and also allowing opportunities such as dose fractionation, which can reduce the effect of sample drift.

Here we describe a direct view of the dynamic processes taking place at the interfaces between nanowires and their catalysts, at the pressures available in ETEM, with aberrationcorrected spatial resolution, and with high temporal resolution. We grow Si nanowires from liquid AuSi, liquid AuAgSi and solid AuAg catalysts, and image the facets and morphology at the growth interface, the structure of the catalyst, the structure of steps and the kinetics of step flow. We find that aberrationcorrected imaging with high image acquisition rate provides useful structural information for both solid and liquid catalysts. However, in making a quantitative comparison between the dynamic information obtained in ETEM and in UHV-TEM, we find distinct differences in the kinetics. We show that the higher gas pressures in ETEM coupled with the greater intensity of beam at the sample modify the catalyst and nanowire surface and alter the growth kinetics. We describe protocols to mitigate the effects of the non-UHV, higher pressure environment. This allows growth to be probed over a higher range of pressures and with atomic resolution to contribute to a mechanistic understanding of nanowire growth. We discuss growth kinetics, corner facets and liquid ordering. Finally, since Si nanowire growth is one of the few systems where ETEM experiments can be compared with those obtained under UHV conditions, we discuss the relevance of these results to interpretation of ETEM data obtained during other dynamic processes.
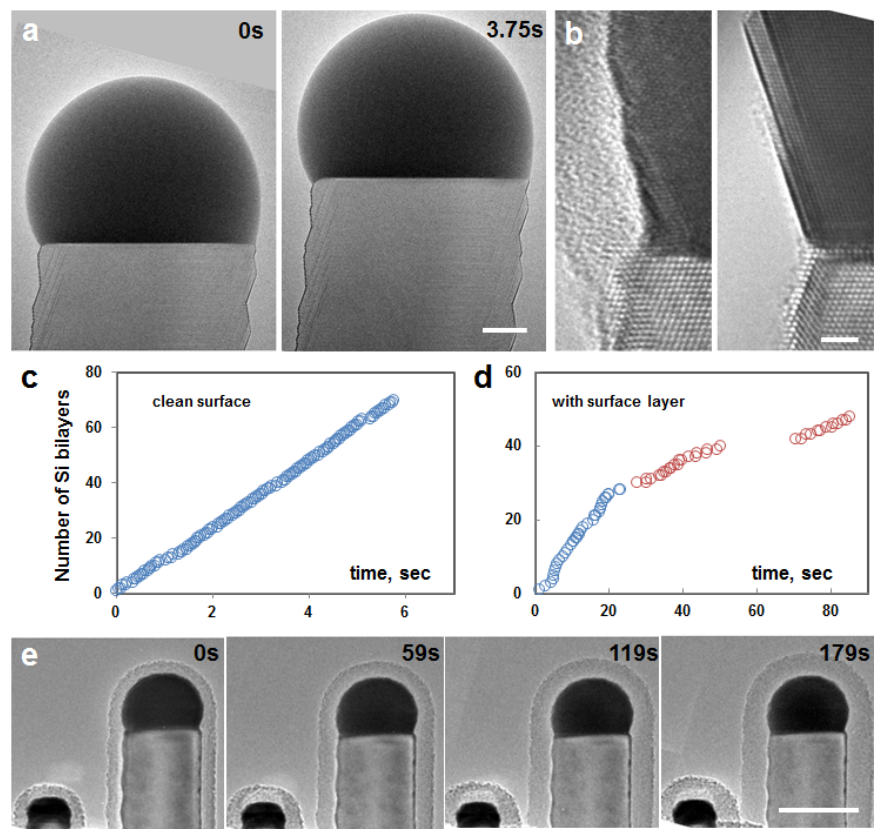

Fig. $1 \mathrm{Si}$ nanowire growth recorded using ETEM at 400 images per second. (a) VLS growth of a $\mathrm{Si}$ nanowire from $\mathrm{AuSi}$ at $500^{\circ} \mathrm{C}$ and $2 \times 10^{-5}$ Torr disilane. The time between images is indicated. Scale bar $=10 \mathrm{~nm}$. Note the clean appearance of the surfaces. (b) Si nanowire formed by VSS from AuAg in the UHVTEM, then transferred to the ETEM and imaged at RT. A surface oxide formed by the air exposure (left image) was removed (right) by focusing the beam on the nanowire without raising T, revealing a faceted catalyst surface. Scale $\mathrm{bar}=2 \mathrm{~nm}$. (c) Growth rate for the nanowire in (a) with clean surface. (d) Growth rate for the nanowire in Fig. S1, ESI $\dagger$ (AuAgSi liquid catalyst, $425^{\circ} \mathrm{C}, 5 \times 10^{-3}$ Torr disilane), with amorphous surface layer. (e) Si nanowire formed by VLS from $\mathrm{Au}$ in the UHVTEM, then transferred to the ETEM and imaged at $550^{\circ} \mathrm{C}$ in $6 \times 10^{-3}$ Torr disilane. No growth occurs; instead an amorphous shell forms. Times indicated in sec. Scale bar $=60 \mathrm{~nm}$. 
A Si nanowire imaged during VLS growth using aberrationcorrected ETEM is shown in Fig. 1a and Movie 1a (ESI +$)$. The growth rate is $3.8 \mathrm{~nm} / \mathrm{sec}$ (Fig. 1c) in this example. UHV-TEM experiments can only probe slower growth (rates achievable are $<1 \mathrm{~nm} / \mathrm{sec}$ at $10^{-5}$ Torr and similar T), so this shows the usefulness of the higher pressure in accessing a greater range of growth kinetics. Overall, nanowires growing in ETEM appear similar to those grown in UHV-TEM - for example, the flat $\mathrm{Si}(111)$ growth interface is visible - but additional information is provided by the higher resolution. The narrow dark contrast visible at the nanowire sidewalls is consistent with a surface layer of $\mathrm{Au}^{20} . \mathrm{Au}$ surface decoration during growth was implied indirectly from ex situ observations. ${ }^{1,21}$ Growth proceeds by rapid flow of steps at the growth interface (Movie 1a). In this particular experiment, each step flows quickly enough that a layer is completed within the time resolution of the movie; there is then a $\sim 0.05 \mathrm{sec}$ pause before the next step flows. Such pause-flow kinetics are expected from models that include the droplet chemical energy and barrier for step nucleation. ${ }^{7,8}$ The temporal and spatial resolution of the image series allows us to provide a lower limit of $\sim 1-2 \mu \mathrm{m} / \mathrm{s}$ on the step flow speed.

In order to obtain such data, it was important to control the growth environment using techniques that we describe below. The nanowire in Fig. 1a appears clean, but earlier experiments yielded images like that in Fig. 1b (left image) or Fig. S1, where amorphous surface layers are visible. We find that, unsurprisingly, such surface layers strongly affect growth kinetics, persisting at elevated temperature and slowing or suppressing VLS and VSS growth during ETEM observations. Presumably the layers are composed of oxides that formed in air on the catalyst surface and prevent the source gas from reaching the catalyst. An example of the growth kinetics measured under such circumstances is shown in Fig. 1d. Note the difference in scale compared to Fig. 1c; growth is $\sim 20 \times$ slower. Also note the irregularity of growth in comparison to Fig. 1c and especially to the highly regular growth curves measured in UHV-TEM. ${ }^{12}$ Surface layers are not fully removed with HF vapor (see ESI $\dagger$ ). It is possible to remove the layer by sputtering using a focused electron beam, a well-known phenomenon $^{22}$ (Fig. 1b). Needless to say, such treatment alters the sample but does provide a clean surface to enable growth to start.

However, once growth does begin, we observe an additional surface layer formation (Fig. 1e, Movie 1b). Energy loss spectroscopy shows that this layer is $\mathrm{SiO}_{\mathrm{x}}$ and does not contain carbon (Fig. S2, ESI $\dagger)$. The formation of this layer requires irradiation (it does not form outside the beam) and the presence of disilane (either during growth, or as a low pressure background due to incomplete pump-down after a previous growth experiment; see below). High temperatures are not required, as we observe layer formation at room temperature $(\mathrm{ESI}+)$. Under UHV conditions ${ }^{1}$ such amorphous surface layers do not form, even under prolonged irradiation at high temperatures in the presence of disilane. We therefore attribute the layer formation to chemical reactions between the electron beam and oxidizing background gases (i.e. water vapor or oxygen) in the ETEM, which is not a UHV system. This reaction mechanism follows the principles of environmental scanning electron microscopy ${ }^{23}$ where water vapor is ionized by the electron beam to form highly reactive species that (in our case) crack the disilane to result in $\mathrm{SiO}_{\mathrm{x}}$. Oxygen may act in the same way. The $300 \mathrm{kV}$ primary beam has a low inelastic cross section with water vapor but the secondary electrons emitted when the primary beam hits the sample have a higher cross section and may be the main cause of the reaction. ${ }^{24}$ Once the shell forms, it slows or prevents $\mathrm{Si}$ from dissolving into the catalyst. Nanowire growth may be slowed or stopped, and growth does not proceed smoothly because the oxide shell pins the catalyst/nanowire interface and prevents it from advancing.

Avoiding this beam effect, with its impact on nanowire growth, requires removal of water vapor or oxygen from the background to achieve UHV levels ( $10^{-10}$ Torr). But even without a UHV system we can mitigate the effects of water vapor or oxygen in ETEM. Water vapor is generally reduced in conventional TEM by condensing it using a cold finger cooled by liquid nitrogen (boiling point $-195.8^{\circ} \mathrm{C}$ / 77.4K). This method can not be used in growth experiments involving disilane since it would condense the disilane (boiling point $-14^{\circ} \mathrm{C} / 259 \mathrm{~K}$ ) along with the water. We used a mixture of dry ice (sublimates at $-78.5^{\circ} \mathrm{C} / 194.7 \mathrm{~K}$ ) and ethanol (melting point $-114^{\circ} \mathrm{C}$ $/ 159 \mathrm{~K}$, boiling point $78.4^{\circ} \mathrm{C} / 351.5 \mathrm{~K}$ ) in order to set the temperature of the cold finger at the sublimation temperature of dry ice. At this temperature the water vapor is still efficiently condensed while a sufficient fraction of the introduced disilane remains in its gaseous state and cracks at the catalyst surface. We finally note that the surface layer can form without deliberately flowing disilane, if there is residual disilane from incomplete pumping after a previous growth experiment (Fig. S2). Flowing $\mathrm{O}_{2}$ to react with and remove residual disilane slowed layer formation. However, completely stopping layer formation required venting the microscope, which effectively removed the residual disilane. This step is dramatic but appears necessary for avoiding layer growth in subsequent experiments.

By using the above procedures it was possible to obtain clean nanowire growth not only with AuSi, as shown in Fig. 1a, but also with catalysts based on metal alloys. Alloy catalysts, such as AuAg and AuAl, can operate in both VLS and VSS modes, with growth by VSS at lower temperatures and VLS at higher temperatures. ${ }^{10,11}$ This is because in the AuAg-Si or AuAl-Si pseudobinary systems the eutectic temperature depends on the alloy composition, allowing suitable temperatures to be selected to obtain either mode.

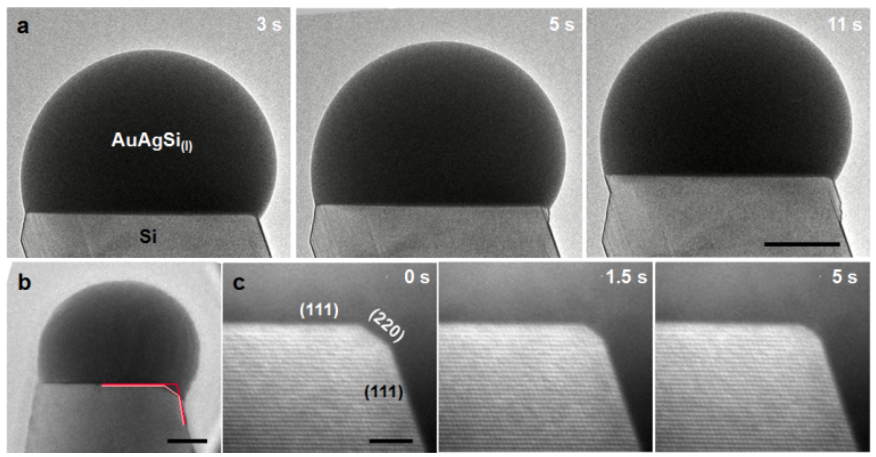

Fig. 2 Si nanowire growth by VLS from AuAgSi. (a) Images recorded during growth of a Si nanowire in ETEM at $500^{\circ} \mathrm{C}$ and $5 \times 10^{-5}$ Torr disilane. Scale bar $=20 \mathrm{~nm}$. (b) Overview of a nanowire with an enclosed facet during growth at at $370^{\circ} \mathrm{C}$ and $1.5 \times 10^{-3}$ Torr disilane. Scale bar $=20 \mathrm{~nm}$. (c) Bright field ETEM image sequence of the truncated corner in (b) during growth. Times indicated in sec. Scale bar $=5 \mathrm{~nm}$.

VLS growth experiments are shown in Fig. 2a and Movie 2a (ESI $\dagger$ ). VLS growth with metal alloy catalysts appears similar to $\mathrm{AuSi}$, with a flat interface between $\mathrm{Si}$ and the liquid across which individual bilayer-height steps flow rapidly. During growth we frequently find junctions between facets at the growth interface, as in Fig. 2b. (Such geometry is especially frequent if the nanowire surface is not completely clean and pins the trijunction during growth.) Aberration correction allows the structure of the nanowire/liquid interface and the edge where facets meet to be resolved during growth, Fig. 2b, c. First note the lack of visible ordering in the liquid adjacent to the solid. Ordering at liquid interfaces has been observed in other materials systems ${ }^{25}$ but is not evident in this liquid catalyst. Also present at the corner is a small truncated section that can be identified as a (220) facet. The dynamical behavior of this facet is shown in Movie $2 b$ $(\mathrm{ESI}+)$. Its size fluctuates as layers are added and removed at the nanowire/catalyst interface. However, the corner remains faceted: we do not observe an atomically sharp configuration during the experiment. Previous observations of dynamic changes in small facets during Si nanowire growth in UHV-TEM lacked the resolution to determine whether the facet is present at all times. The presence or 
absence of a higher index facet at an edge where larger facets meet is commonly understood via the Wulff construction. However, the Wulff construction is based on an equilibrium crystal, and in principle does not apply during growth. Instead, it has been suggested that small facets should be sensitive to the supersaturation of $\mathrm{Si}$ in the liquid and should therefore grow and shrink with the nucleation of steps. ${ }^{12}$ The observation in Fig. 2c is consistent with the model.

The importance of this observation lies in understanding nucleation energetics for Si growth. During growth, new layers are expected to nucleate at the periphery of the (111) growth plane. Creating a nucleus at the periphery also extends the facet that bounds the growth plane. Thus, the energy barrier for nucleation will depend on which facets bound the growth plane. Observations such as those in Movie 2 that can measure fluctuations in bounding facets may provide a basis for improved calculations of nucleation energetics. ${ }^{26}$

VSS growth is an important mode that is well-known in several materials systems ${ }^{14}$; the growth species is assumed to diffuse through the solid particle to the growth front. VSS growth with metal alloy catalysts in aberration corrected ETEM is shown in Fig. 3a, c for the example of Si growth from AuAg. For comparison, Fig. 3b, d shows

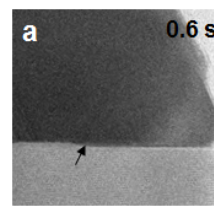

b

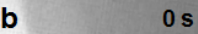

1
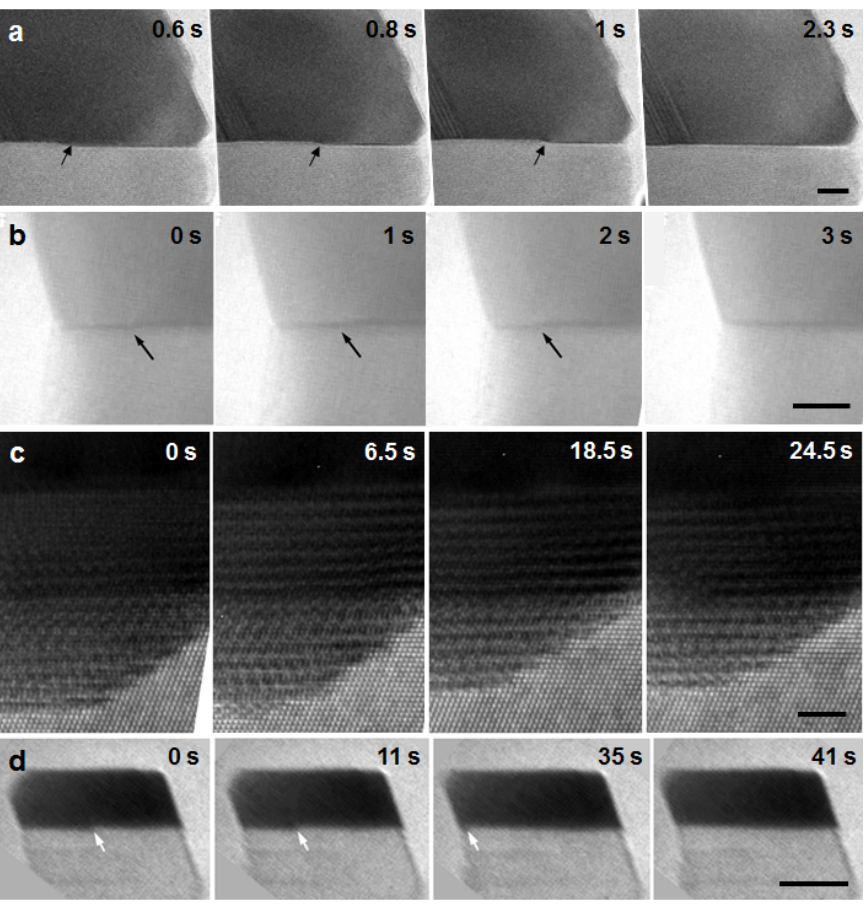

Fig. $3 \mathrm{Si}$ nanowire growth, interface structure and step flow at solid $\mathrm{AuAg} / \mathrm{Si}$ interfaces. (a) Image sequence showing a Si step with single Si bilayer moving along the interface with $\mathrm{AuAg}$ at $450^{\circ} \mathrm{C}$ and $5 \times 10^{-4}$ disilane in ETEM. Scale bar $=5 \mathrm{~nm}$. (b) Image sequence showing a Si step (arrowed) with single Si bilayer moving along the interface with $\mathrm{AuAg}$ at $483^{\circ} \mathrm{C}$ and $9.3 \times 10^{-6}$ Torr disilane in UHV-TEM. Scale bar $=$ $5 \mathrm{~nm}$. (c) Image sequence showing dynamics at an inclined interface between solid $\mathrm{AuAg}$ and $\mathrm{Si}$ at $380^{\circ} \mathrm{C}$ and $1.5 \times 10^{-3}$ Torr disilane in ETEM. Rearrangement occurs by the motion of units of height $3 \mathrm{Si}$ bilayers / $4 \mathrm{AuAg}$ layers. The longer period contrast in the AuAg is a moiré effect due to overlap with $\mathrm{Si}$ in projection. Scale bar $=3 \mathrm{~nm}$. (d) Image sequence showing a Si step (arrowed) with triple Si bilayers moving along the interface with $\mathrm{AuAg}$ at $470^{\circ} \mathrm{C}$ and $3 \times 10^{-5}$ Torr disilane in UHV-TEM. The step flows in a [211] direction on the (111) plane. The contrast lower down is caused by twinning in the Si due to temperature changes during the experiment. Scale bar $=20 \mathrm{~nm}$.

growth under UHV conditions. The orientation relation between $\mathrm{Si}$ and $\mathrm{AuAg}$ lattices and the overall truncated octahedron shape of the solid AuAg are consistent between ETEM and UHV TEM (Movie 3,
ESI $\dagger) .{ }^{10}$ Interface dynamics involve step flow in both cases but there are intriguing differences in step geometry. The steps in Fig. 3a, b are a single Si bilayer in height $(0.3 \mathrm{~nm})$ but those in Fig. $3 \mathrm{c}, \mathrm{d}$ are triple height, composed of three Si bilayers $(0.9 \mathrm{~nm})$. We observe flow of single bilayer steps in ETEM if the surface is clean, but triple bilayer steps in the presence of the surface layer described above. Since the surface layer prohibited or slowed dissolution of Si into the catalyst, the process taking place could more accurately be described as interface rearrangement rather than growth of $\mathrm{Si}$. In UHV-TEM, single height steps were common and triple height steps (Fig. 3d) were observed only for a small diameter nanowire that grew slowly even at higher pressure. We suggest that triple height steps may be preferred during, for example, rearrangement between solid catalyst and $\mathrm{Si}$, because of the good lattice match between three Si bilayers and four $\mathrm{AuAg}$ planes (the mismatch between $\mathrm{AuAg}$ and $\mathrm{Si}$ is $24.9 \%$ ). ${ }^{10}$ The mismatch also leads to a periodic structure in-plane along the interface (Fig. S3a, ESI $\dagger$ ) with one dislocation every $0.95 \mathrm{~nm}$.

VSS growth is expected to show different kinetics compared to VLS since atomic level details of the solid/solid interface such as step height and local strain fields should influence the growth mechanism. Steps at the solid $\mathrm{AuAg} / \mathrm{Si}$ interface move relatively slowly during growth, compared to the rapid VLS step motion in Fig. 2a. The slow VSS step dynamics has been explained through models ${ }^{11,13}$ where the low solubility of $\mathrm{Si}$ in the solid catalyst makes it energetically favorable for $\mathrm{Si}$ to precipitate at the growth interface as soon as it arrives from the gas phase. Thus, Si adds gradually to an existing step, and nucleation of a new step takes place shortly after the previous layer has completed. These models do not include atomic level details of the interface, but such details are expected to affect step motion. We observe that steps do not flow smoothly. In Movie 3, motion is jerky and pinned at some points, similar to observations in other VSS systems ${ }^{8,10,13}$ where pinning appears to be related to interfacial dislocations.

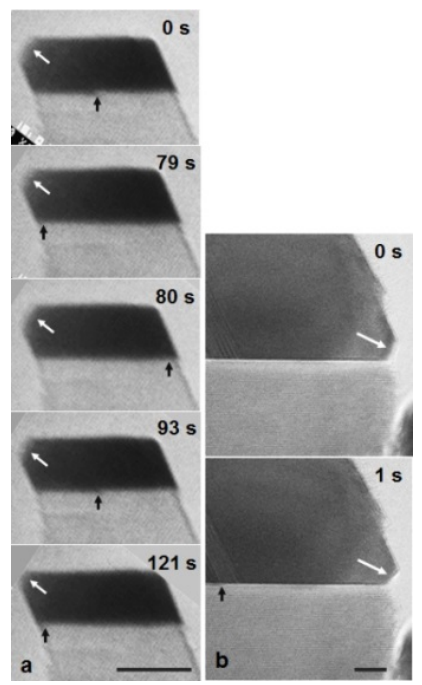

Fig. 4 Rearrangement of the solid catalyst surface. (a) Change in catalyst shape (white arrows) during VSS growth from AuAg at $470^{\circ} \mathrm{C}$ and $3 \times 10^{-5}$ Torr disilane in UHV-TEM. The size of the upper left facet varies during step flow; $1 \frac{1}{2}$ cycles are shown. Scale bar $=20 \mathrm{~nm}$. (b) Change in catalyst shape during VSS growth from AuAg in ETEM at $450^{\circ} \mathrm{C}$ and $5 \times 10^{-4}$ Torr disilane. Scale bar $=5 \mathrm{~nm}$.

We finally note intriguing changes in the overall catalyst shape during VSS growth experiments. Fig. 4a and Movie 3d hint that during UHV growth, the catalyst shape changes periodically as steps flow during growth. A truncated corner fills during step flow then the facet size jumps as a new step starts. In ETEM, rearrangement of the solid catalyst surface is also visible (Fig. S3, S4 and Movie 4a, 4b, Fig. 
4b) but without the clear synchronization with interfacial step flow seen in UHV-TEM. We suggest that the nanoparticle as a whole may rearrange as steps flow to satisfy considerations of energy minimization. ${ }^{27}$

In conclusion, we have shown that aberration-corrected ETEM can reveal detailed features of step flow kinetics, facets at the nanowire/catalyst interface, and rearrangement of the solid catalyst surface. High resolution allows atomic configurations to be characterized, including individual events during the flow of steps. However, surface layers form in ETEM and the kinetics for $\mathrm{Si}$ incorporation differ compared to observations made during growth under UHV conditions. In ETEM, we suggest that surface layers form through reaction of the electron beam with residual oxygen or water vapor in the microscope vacuum. It is therefore essential to include beam effects when interpretating growth kinetics obtained in a non-UHV system. The detailed knowledge that already exists for nanowire growth under UHV is useful in disentangling beam effects and side reactions. But in other materials systems, where conclusions on reaction mechanisms are based on ETEM, we need to consider whether analogous side reactions may have significant effects. We have proposed a method of achieving clean Si nanowire growth in a non-UHV ETEM. It is possible that analogous technique may be useful for other growth materials. With this type of protocol, aberration corrected ETEM has exciting prospects for understanding growth mechanisms at the atomic level.

This work was supported by the National Science Foundation under Grants No. DMR-0606395 and 0907483, the Ministry of Science and Technology R.O.C. under Grant No. NSC-1012112-M-009-021-MY3 and MOST-104-2112-M-009-015-MY3, the Center for Interdisciplinary Science under the MOE-ATU project for NCTU, ERC Grant 279342: InSituNANO, and the Center for Functional Nanomaterials, Brookhaven National Laboratory, which is supported by the US Department of Energy, Office of Basic Energy Sciences, under contract DE-AC02$98 \mathrm{CH} 10886$.

\section{Notes and references}

1 F. M. Ross, Rep. Prog. Phys., 2010, 73114501.

2 F. M. Ross, J. Tersoff, and M. C. Reuter, Phys. Rev. Lett., 2005, 95, 146104.

3 J. B. Hannon, S. Kodambaka, F. M. Ross and R. M. Tromp, Nature, 2006, 440, 69 .

4 S. Kodambaka, J. Tersoff, M. C. Reuter and F. M. Ross, Phys. Rev. Lett., 2006, 96, 96105.

5 P. Madras, E. Dailey and J. Drucker, Nano Lett., 2009, 9, 3826.

6 B. A. Wacaser, M. C. Reuter, M. M. Khayyat, C.-Y. Wen, R. Haight, S. Guha and F. M. Ross, Nano Lett., 2009, 9, 3296.
7 S. Kodambaka, J. Tersoff, M. C. Reuter and F. M. Ross, Science, 2007, 316, 729.

8 C.-Y. Wen, M. C. Reuter, J. Tersoff, E. A. Stach and F. M. Ross, Nano Lett., 2010, 10, 514.

9 S. Hofmann, R. Sharma, C. T. Wirth, F. Cervantes-Sodi, C. Ducati, T. Kasama, R. E. Dunin-Borkowski, J. Drucker, P. Bennett and J. Robertson, Nature Mater., 2008, 7, 372.

10 Y.-C. Chou, C.-Y. Wen, M. C. Reuter, D. Su, E. A. Stach and F. M. Ross, ACS Nano, 2012, 6, 6407.

11 C.-Y. Wen, M. C. Reuter, J. Bruley, J. Tersoff, S. Kodambaka, E. A. Stach and F. M. Ross, Science, 2009, 326, 1247.

12 C.-Y. Wen, J. Tersoff, K. Hillerich, M. C. Reuter, J. H. Park, S. Kodambaka, E. A. Stach and F. M. Ross. Phys. Rev. Lett., 2011, 107, 025503.

13 C.-Y. Wen, J. Tersoff, M. C. Reuter, E. A. Stach, and F. M. Ross, Phys. Rev. Lett., 2010, 105, 195502.

14 K. Hiruma, M. Yazawa, T. Katsuyama, K. Ogawa, K. Haraguchi, M. Koguchi, H. Kakibayashi, J. Appl. Phys., 1995, 77, 447; T. I. Kamins, R. S. Williams, D. P. Basile, T. Hesjedal, J. S. Harris, J. Appl. Phys., 2001, 89, 1008; A. I. Persson, M. W. Larsson, S. Stenström, B. J. Ohlsson, L. Samuelson, L. R. Wallenberg, Nat. Mater., 2004, 10, 677.

15 J. Hu, T. W. Odom and C. M. Lieber, Acc. Chem. Res., 1999, 32, 435.

16 C. B. Duke, ed., Surface Science: The First Thirty Years (Elsevier, Amsterdam, 1994).

17 A. M. Molenbroek, S. Helveg, H. Topsøe, and B. S. Clausen, Top. Catal., 2009, 52, 1303.

18 H. Yoshida, Y. Kuwauchi, J. R. Jinschek, K. Sun, S. Tanaka, M. Kohyama, S. Shimada, M. Haruta, and S. Takeda, Science, 2012, 335, 317; Y.-C. Chou, M. C. Reuter, E. A. Stach, and F. M. Ross, Microsc. Microanal. 2012, 18(S2), 1084-1085; F. Panciera, Y.-C. Chou, M. C. Reuter, D. Zakharov, E. A. Stach, S. Hofmann, F. M. Ross, Nat. Mater., 2015, 14, 820 .

19 D. Contarato, P. Denes, D. Doering, J. Joseph, and B. Krieger, Physics Procedia, 2013, 37, 1504.

20 T. Kamino, T. Yaguchi, M. Tomita, and H. Saka, Philos. Mag. A, 1997, 75, 105 .

21 C. Wiethoff, F. M. Ross, M. Copel, M. Horn-von Hoegen and F.-J. Meyer zu Heringdorf, Nano Lett., 2008, 8, 3065.

22 G. S. Chen, C. B. Boothroyd, and C. J. Humphreys, Philos. Mag. A, 1998, 78, 491; N. Jiang, Rep. Prog. Phys., 2016, 79, 016501.

23 D. J. Stokes, Principles and practice of variable pressure / environmental scanning electron microscopy, Wiley, 2008.

24 J. H. Miller, W. E. Wilson, S. T. Manson and M. E. Rudd, J. Chem. Phys., 1987, 86, 157.

25 M. Baram, D. Chatain, and W. D. Kaplan, Science, 2011, 332, 206; J. M. Howe and H. Saka, MRS Bull., 2004, 29, 951; U. Dahmen, Phys. Rev. Lett., 1997, 78, 471.

26 Y. Yang, M. Asta and B. B. Laird, Phys. Rev. Lett., 2013, 110, 096102.

27 J. Tersoff, A. W. Denier van der Gon, and R. M. Tromp, Phys. Rev. Lett., 1993, 70, 1143. 


\title{
Electronic Supplementary Information
}

\section{Nanowire growth kinetics in aberration corrected environmental transmission electron microscopy}

\author{
Yi-Chia Chou ${ }^{\mathrm{a}, \mathrm{b}, \mathrm{c}}$, Federico Panciera ${ }^{\mathrm{c}, \mathrm{d}}$, Mark C. Reuter ${ }^{\mathrm{c}}$, Eric A. Stach ${ }^{\mathrm{a}}$ and Frances M. Ross ${ }^{\mathrm{c}}$ \\ ${ }^{a}$ Center for Functional Nanomaterials, Brookhaven National Laboratory, Upton, NY, U.S.A. \\ ${ }^{b}$ Department of Electrophysics, College of Science, National Chiao Tung University, Hsinchu, Taiwan. \\ ${ }^{c}$ IBM Research Division, T. J. Watson Research Center, Yorktown Heights, NY, U.S.A. \\ ${ }^{d}$ Engineering Department, University of Cambridge, Cambridge, United Kingdom.
}

\section{Details of experimental procedure}

The experiments were carried out in a Cs-corrected FEI Titan 80-300 ETEM that has capabilities for introducing multiple gases through mass flow controllers into a mixing manifold that is connected to the sample region of the microscope. ${ }^{1}$ The base pressure of this instrument is $10^{-7}$ Torr in standard mode, but $2-4 \times 10^{-6}$ Torr (without cold finger) in ETEM mode, where some pumps are valved off to allow higher pressures at the sample region. The base pressure is determined by air arriving through the sample rod o-ring, so includes $\mathrm{N}_{2}, \mathrm{O}_{2}, \mathrm{H}_{2} \mathrm{O}$ and other minor components. We examined Si nanowire growth, using Au for experiments on the VLS growth mode and AuAg alloys for experiments on both VSS and VLS modes. Pure disilane was used as the precursor and was flowed over Si samples heated to temperatures in the range $350-450^{\circ} \mathrm{C}$. The maximum disilane pressure was $5 \times 10^{-3}$ Torr with a measured flow rate of $0.2 \mathrm{sccm}$. On heating and exposure to disilane, image series were recorded at Gatan Ultrascan CCD with 2 frames per second and Gatan K2-IS CCD with 400 frames per second for in situ movie recording.

Rather than reacting the disilane with catalyst particles placed directly on the substrate, we imaged the dynamics at the tips of pre-grown nanowire "stubs". These nanowire samples were prepared from substrates cut into the TEM sample size of $3 \mathrm{~mm} \times 500 \mu \mathrm{m} \times 500 \mu \mathrm{m}$. Either $\mathrm{Au}$ or $\mathrm{Au}+\mathrm{Ag}$ sequentially was deposited on the sample surface as a continuous film. The samples were heated to agglomerate the metal into droplets of diameter 20-50nm. The quoted AuAg compositions are approximate and based on the deposited Au and Ag thickness. There is a solid solution at all compositions, but each particle could have a somewhat different composition depending on diffusion of Au and Ag during agglomeration ${ }^{2}$. The exact composition is not critical for interpretation, since Ref. 2 shows little dependence of growth kinetics on composition; the major effect of composition is to set the eutectic temperature. After catalyst agglomeration, disilane was flowed to grow Si nanowires to a length of 200-500nm. The catalyst deposition and nanowire growth were carried out in a UHV-TEM. ${ }^{2}$ Since growth took place under observation, we could ensure that the resulting nanowires had suitable length, diameter and density. Experimental details of the UHV-TEM growth are provided elsewhere. ${ }^{2}$ The samples were transferred through air and cleaned by HF-vapor etching immediately before loading into the ETEM. Growth in ETEM is at different temperature and pressure, leading to a transient change in the nanowire diameter (see Section 3 below for an image).

\section{Effect of surface layer on growth morphology and step flow kinetics: VLS}

Fig. S1(a) shows a nanowire at lower magnification after the end of a typical disilane exposure without the surface cleaning protocol described in the text, and Fig. S1(b) shows growth via step flow. The segment that has grown in the ETEM is visible above a narrower region. This narrowing of the nanowire is commonly observed in the ETEM samples. It is not present when the samples are first grown, and is a consequence of the processing that is necessary between making the samples and carrying out ETEM. Cooling the sample after growth, solidifying the catalyst, exposing to air, etching with HF, and reheating in the ETEM result in a liquid droplet and nanowire of slightly altered geometry. because the growth temperature in the ETEM is slightly different from that in the UHVTEM, changing the amount of dissolved $\mathrm{Si}$ in the catalyst; or because the nanowire sidewalls have oxidized, changing their surface energy and hence the contact angle at the trijunction. 
During growth, the surface layer appears to pin the trijunction and the growth front does not advance uniformly. The growth interface can show facets of (111) and (220), and the addition of ledges is slow and not regularly timed, presumably related to the overall morphology and surface condition of the nanowire during growth in the ETEM.
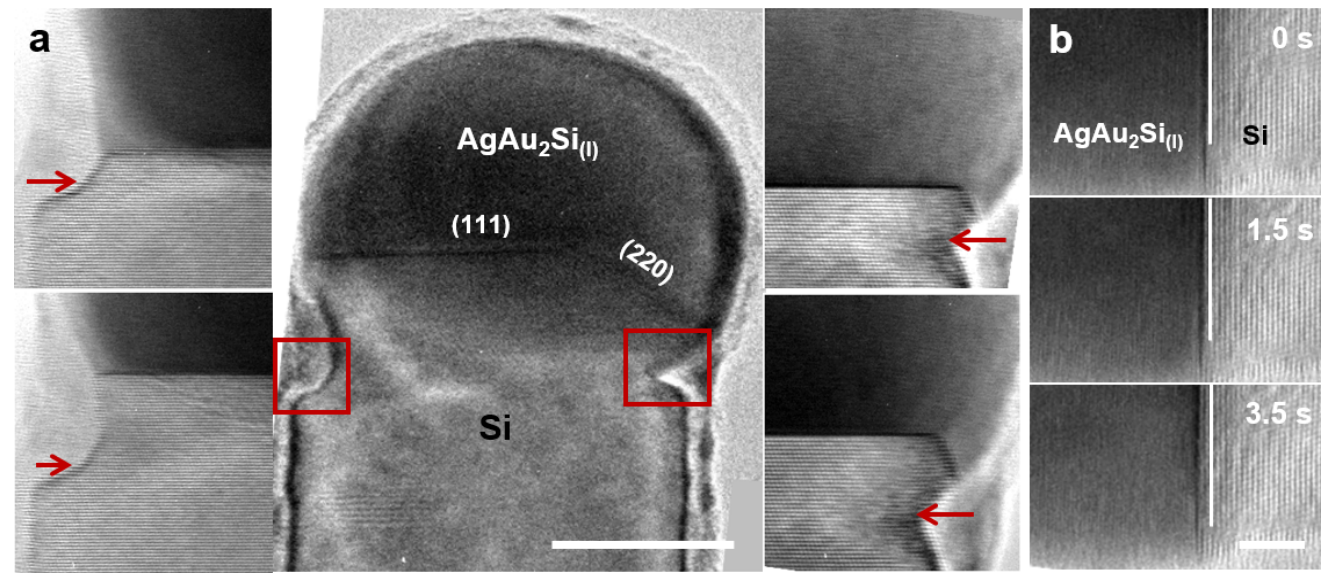

Fig. S1 Si nanowire growth by VLS in ETEM from AuAgSi at $425^{\circ} \mathrm{C}$ with $5 \times 10^{-3}$ Torr $\mathrm{Si}_{2} \mathrm{H}_{6}$. (a) A lower magnification image of another Si nanowire. The interface before growth is indicated by red arrows at the trijunctions. The inset images show stages of growth at both trijunctions. Scale bar $=30 \mathrm{~nm}$. (b) Images recorded during growth at $425^{\circ} \mathrm{C}$ with $5 \times 10^{-3}$ Torr disilane. During the sequence, Si bilayers $(0.31 \mathrm{~nm})$ flow across the interface within one frame. Scale bar $=4 \mathrm{~nm}$.

\section{Surface layer formation in ETEM}

We observed two types of surface layer; each prevents or slows incorporation of Si from disilane into the catalyst and nanowire.

(1) An oxide forms during air exposure of the sample before loading, and persists even with HF vapor treatment. Removal of this layer can be accomplished by focusing the electron beam onto the sample surface. Fig S2(a) shows progressive removal, allowing facets to form in the solid parts of the catalyst and Si to incorporate into the catalyst and transform solid AuAg to solid plus liquid (AuAgSi).

(2) A surface layer of silicon oxide forms under the electron beam, Fig S2(b-e). The example shown here illustrates a surface layer forming on a sample even without deliberate disilane, but with residual disilane present from a previous growth experiment. The formation rate is slowed by introducing $\mathrm{O}_{2}$ to react with and remove disilane; venting the microscope effectively removed residual disilane. 

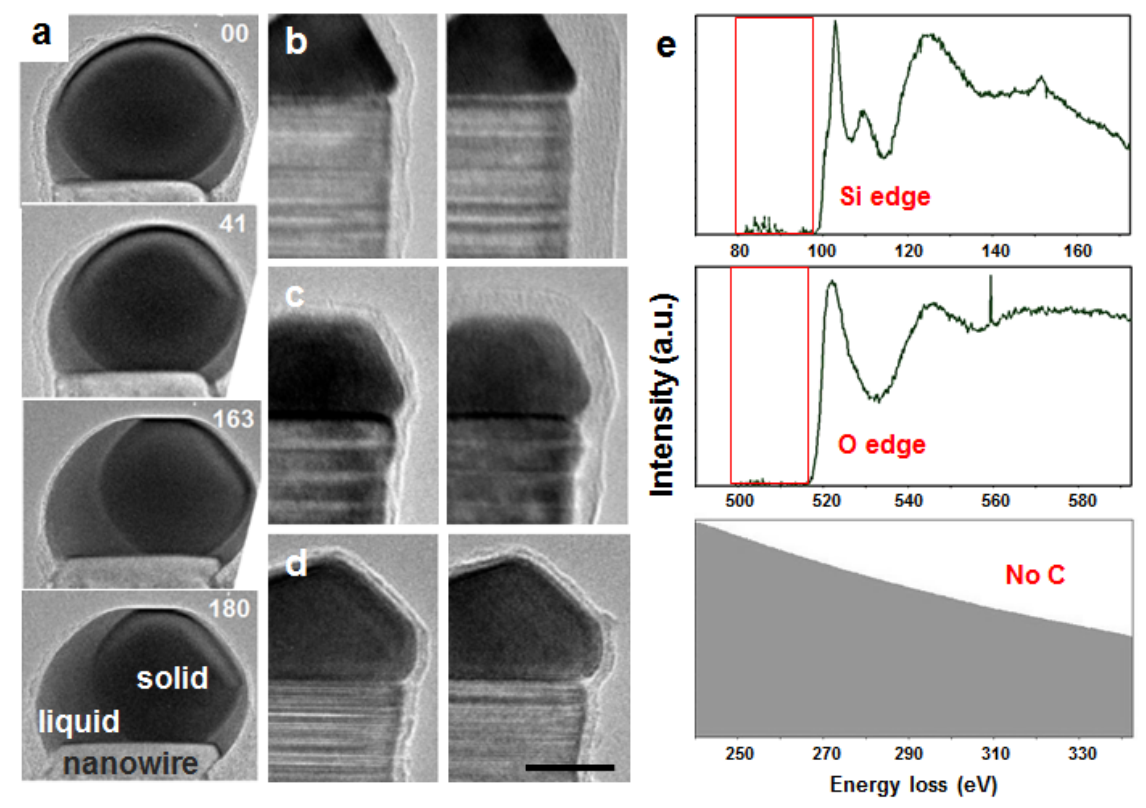

Fig. S2 (a) Surface layer removal using a focused electron beam shower at $550^{\circ} \mathrm{C}$ and $9.5 \times 10^{-3}$ Torr disilane. From the AuAg-Si phase diagram we expect the catalyst to be fully liquid at temperatures over $525^{\circ} \mathrm{C}$. The reaction is slowed because $\mathrm{Si}$ can not add easily to the droplet until the surface is cleaned. The time is shown in seconds. (b) The rate of surface oxide layer formation on a GaP nanowire is $1 \mathrm{~nm} / \mathrm{min}$ in ETEM at RT with residual disilane. (c) The rate slows to $0.5 \mathrm{~nm} / \mathrm{min}$ after flowing $\mathrm{O}_{2}$ to remove residual disilane. (d) No surface layer growth after the microscope was vented to remove residual disilane. Different regions of the sample were imaged in each case. Scale bar $=25 \mathrm{~nm}$. (e) EELS results showing that the surface layer is $\mathrm{SiO}_{\mathrm{x}}$ and does not contain carbon. A GaP nanowire was used for this analysis so that no Si was present in the sample.

\section{Effect of surface layer on growth kinetics: VSS; catalyst surface rearrangement}

Fig. S3(a) shows the structure of the AuAg/Si catalyst/nanowire interface after VSS growth from AuAg then imaged post-growth at room temperature. Note the periodic structure visible along the interface due to the lattice mismatch (all AuAg compositions have FCC structure and almost identical lattice parameters.) Steps at this interface are shown in the manuscript. Fig S3(b) shows interface rearrangements observed on heating samples such as in (a) in disilane in the ETEM. The rate of net addition of Si is small, and may even be negative: Fig. S3(c) and Movie 5 show a backwards movement of a VSS catalyst/nanowire interface as Si from the nanowire dissolves into the catalyst on heating. Since we expect growth rates of a few $\mathrm{nm}$ per minute ${ }^{2}$, it appears that (as was the case for VLS growth), the incorporation rate of $\mathrm{Si}$ is slower than expected for the temperatures and pressures used.

Fig. S4(a, b) shows the catalyst shape changing as the catalyst/nanowire interface rearranges during growth with ledges moving at the surface. 


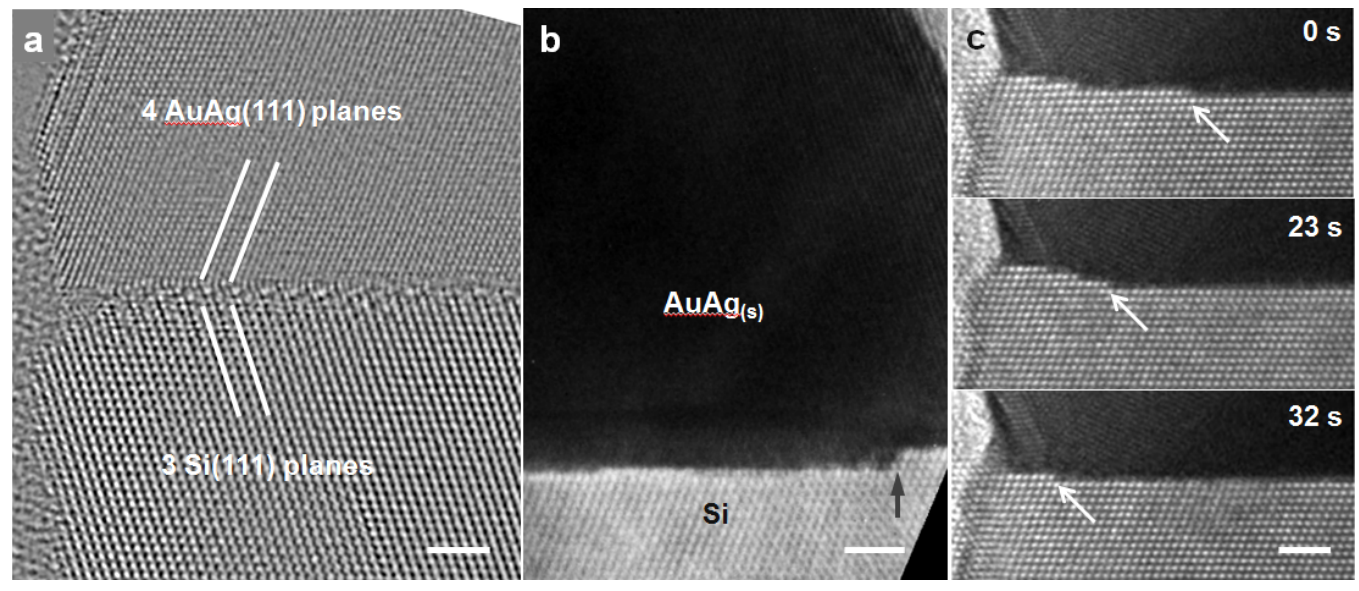

Fig. S3 Structure and ledge flow at solid AuAg/Si interfaces. (a) Post-growth aberration corrected image showing the solid AuAg/VSS-grown Si nanowire interface; exit wave reconstruction recorded before growth in ETEM. The match between 3 $\mathrm{Si}(111)$ planes and $4 \mathrm{AuAg}(111)$ planes is shown. (b) ETEM image showing flow of triple bunched Si bilayers during rearrangement of solid AuAg catalyst and $\mathrm{Si}$ at $380{ }^{\circ} \mathrm{C}$ and $1.5 \times 10^{-3}$ Torr disilane. (c) Sequence of ETEM images showing a backwards movement of Si from a VSS catalyst/nanowire interface during heating at $400{ }^{\circ} \mathrm{C}$. Scale bars $=2 \mathrm{~nm}$.
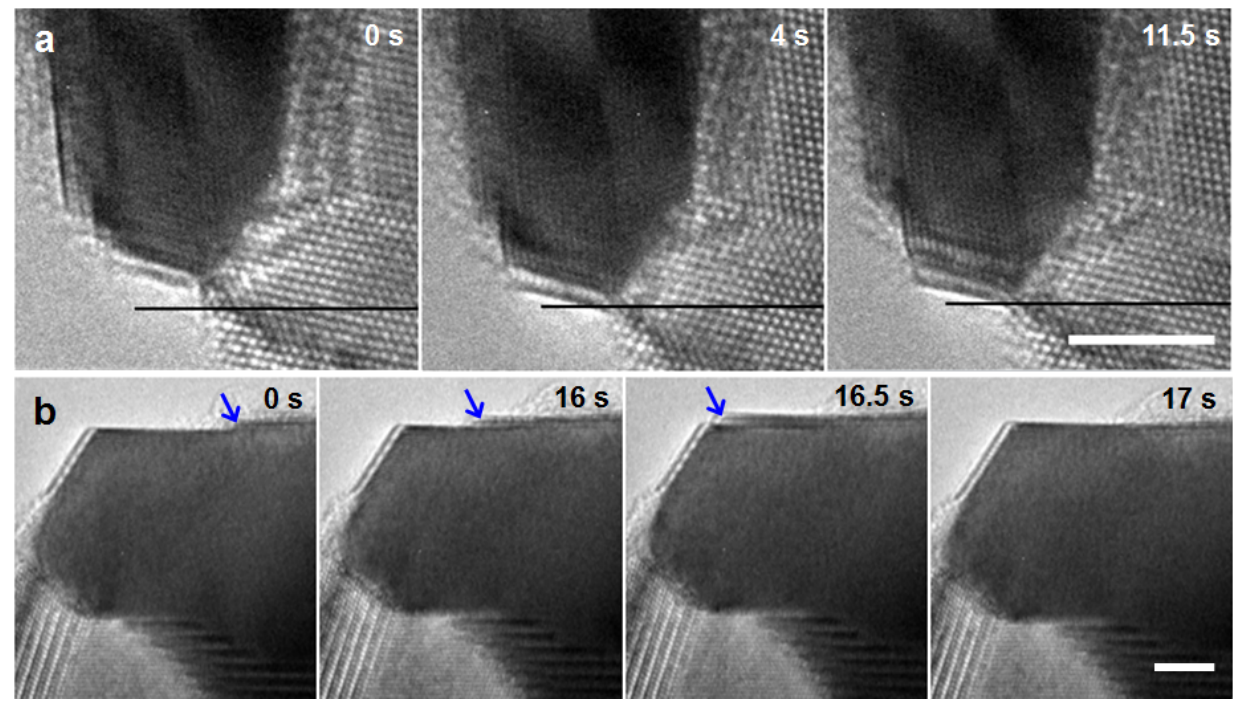

Fig. S4 Ledge flow on solid catalyst sidewalls. (a) Rearrangement of the side wall of a solid AuAg catalyst in ETEM at $380^{\circ} \mathrm{C}$ and $1.5 \times 10^{-3}$ Torr disilane. Black line is for reference. (b) Ledge flow on solid AuAg catalyst in ETEM at $380^{\circ} \mathrm{C}$ and $1.5 \times 10^{-3}$ Torr disilane. Scale bars $=5 \mathrm{~nm}$.

\section{Movie captions}

Movie 1 (Fig. 1a, Fig. 1d): 


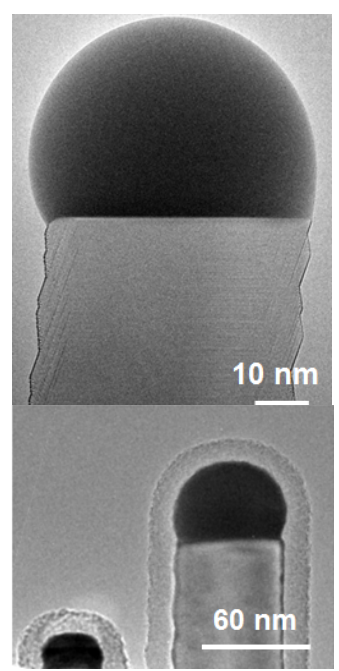

a, (Fig. 1a) ETEM movie showing VLS growth of a Si nanowire with clean surface from AuSi in ETEM at $500{ }^{\circ} \mathrm{C}$ with $5 \times 10^{-5}$ Torr disilane, $2 \times$ speed.

b, (Fig. 1d) ETEM movie showing amorphous shell formation on a pre-grown Si nanowire at $550^{\circ} \mathrm{C}$ and $6 \times 10^{-3}$ Torr disilane. The amorphous shell becomes thicker with time. The movie is at $10 \times$ speed.

Movie 2 (Fig. 2a, Fig. 2c-e, Fig. S2c):

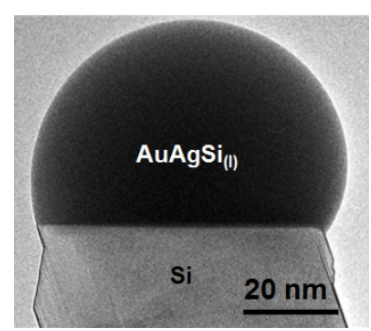

a, (Fig. 2a): ETEM movie showing VLS growth of a Si nanowire with clean surface from AuAgSi in ETEM at $500{ }^{\circ} \mathrm{C}$ with $5 \times 10^{-5}$ Torr disilane, actual speed.

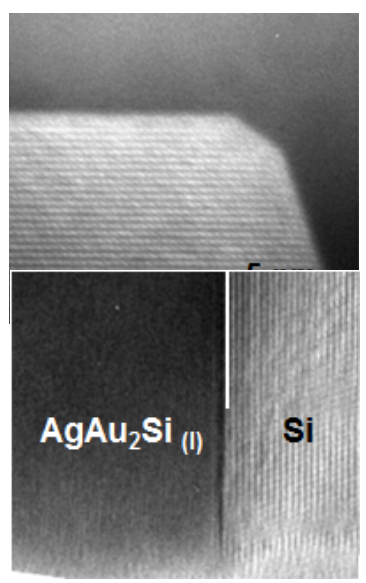

b, (Fig. 2c): Changes in the configuration at the truncated corner between two $\{111\}$ planes, during VLS Si nanowire growth from AUAgSi at $370^{\circ} \mathrm{C}$ and $1.5 \times 10^{-3}$ Torr disilane in ETEM, $2.5 \times$ speed. The size of the truncation fluctuates as layers are added and removed at the Si/AuSi interface.

c, (Fig. S2b): ETEM movie showing VLS growth of a Si nanowire from AuAgSi at $425^{\circ} \mathrm{C}$ and $5 \times 10^{-3}$ Torr $\mathrm{Si}_{2} \mathrm{H}_{6}, 2.5 \times$ speed. VLS growth occurs by rapid single Si bilayer addition.

Movie 3 (Fig. 3a, b, c, and d):

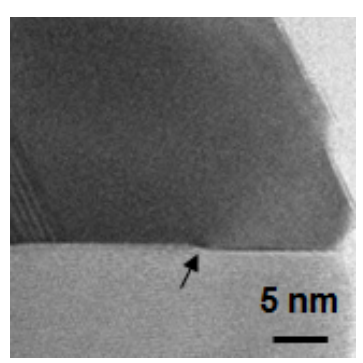

a, (Fig. 3a): ETEM movie showing flow of a single Si bilayer during VSS growth of Si nanowire with clean surface from solid AuAg catalyst, $5 \times 10^{-4}$ Torr disilane at $450^{\circ} \mathrm{C}$, actual speed. 

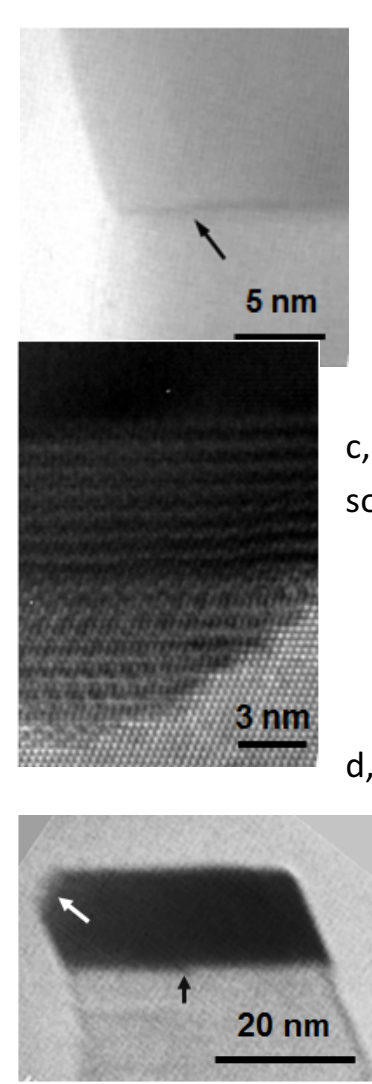

d, (Fig. 3d): UHV-TEM movie showing VSS growth of a Si nanowire from AuAgSi at $470^{\circ} \mathrm{C}$ and $3 \times 10^{-5}$ Torr disilane, $3 \times$ speed. Si ledges move along the interface between catalyst and $\mathrm{Si}$ nanowire in the [211] direction of the (111) plane. The upper left corner of the catalyst, a $\{100\}$ plane, varies in size during growth.

c, (Fig. 3c): ETEM movie showing flow of triple bunched Si bilayers during rearrangement of solid AuAg catalyst and Si on exposure to $1.5 \times 10^{-3}$ Torr disilane at $380^{\circ} \mathrm{C}, 2.5 \times$ speed.

Movie 4 (Fig. S4, Fig. 4b):

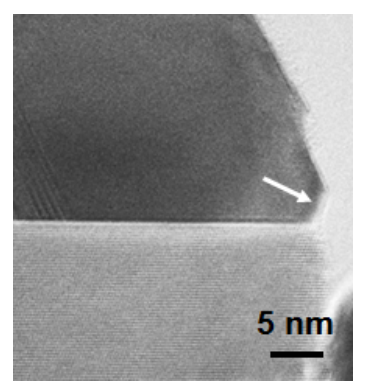

a, (Fig. 4b) Rearrangement of AuAg solid catalyst during growth at $450^{\circ} \mathrm{C}$ and $5 \times 10^{-4} \mathrm{Torr}$ disilane, shown at actual speed.

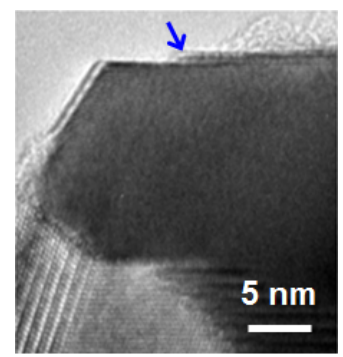

b. (Fig. S4) ETEM movie showing ledge flow on solid catalyst during VSS growth on exposure to $1.5 \times 10^{-3}$ Torr disilane at $380^{\circ} \mathrm{C}, 8 \times$ speed.

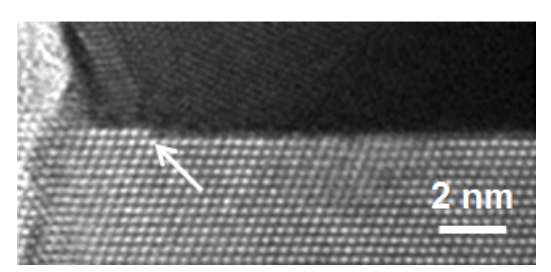

ETEM movie showing retraction of the Si/VSS catalyst interface at steady conditions $\left(400{ }^{\circ} \mathrm{C}\right)$ and without surface treatment. This sequence is extracted from a longer movie of etching lasting 2.5 minutes. The movie is at $4 \times$ speed.

\section{References}


1 Y.-C. Chou, M. C. Reuter, E. A. Stach, and F. M. Ross, Microsc. Microanal. 2012, 18(S2), 1084-1085; F. Panciera, Y.-C. Chou, M. C. Reuter, D. Zakharov, E. A. Stach, S. Hofmann, F. M. Ross, Nat. Mater., 2015, 14, 820.

2 Y.-C. Chou, C.-Y. Wen, M. C. Reuter, D. Su, E. A. Stach and F. M. Ross, ACS Nano, 2012, 6, 6407. 